\title{
Formation and Destruction of Silicon Monoxide in SN 1987A
}

\section{Citation}

Liu, Weihong, and A. Dalgarno. 1996. "Formation and Destruction of Silicon Monoxide in SN 1987A." The Astrophysical Journal 471 (1): 480-84. https://doi.org/10.1086/177982.

\section{Permanent link}

http://nrs.harvard.edu/urn-3:HUL.InstRepos:41397475

\section{Terms of Use}

This article was downloaded from Harvard University's DASH repository, and is made available under the terms and conditions applicable to Other Posted Material, as set forth at http:// nrs.harvard.edu/urn-3:HUL.InstRepos:dash.current.terms-of-use\#LAA

\section{Share Your Story}

The Harvard community has made this article openly available.

Please share how this access benefits you. Submit a story.

\section{Accessibility}




\title{
FORMATION AND DESTRUCTION OF SILICON MONOXIDE IN SN 1987A
}

\author{
WeiHong LiU AND A. Dalgarno \\ Harvard-Smithsonian Center for Astrophysics, Cambridge, MA 02138 \\ Received 1996 March 21; accepted 1996 May 31
}

\begin{abstract}
We present a new chemistry model for the silicon monoxide molecules in SN 1987A. It incorporates an enhanced rate of formation by radiative association and an enhanced rate of destruction through charge transfer between $\mathrm{SiO}$ and $\mathrm{Ar}^{+}$and $\mathrm{Ne}^{+}$, which is more efficient than the energetic electron impact ionization and dissociation in a previous model. The conclusion is unchanged that the $10^{-4}-10^{-3} M_{\odot}$ of $\mathrm{SiO}$ observed in SN 1987A can be produced by chemistry models in which there is no microscopic mixing of helium into the silicon-oxygen region of the ejecta.

Subject headings: molecular processes - supernovae: individual (SN 1987A)
\end{abstract}

\section{INTRODUCTION}

Infrared emission from vibrationally excited silicon monoxide molecules was observed in the ejecta of SN 1987A as early as 160 days after the supernova explosion (Aitken et al. 1988) and could no longer be detected after 578 days (Roche et al. 1989). The fundamental emission spectra of $\mathrm{SiO}$ have been analyzed by Liu \& Dalgarno (1994). By accounting for optical depth effects on the emission and non-LTE effects on the vibrational level populations, Liu \& Dalgarno (1994) have inferred $10^{-4}-10^{-3} M_{\odot}$ for the mass of $\mathrm{SiO}$ in the ejecta.

The chemical processes that form and destroy $\mathrm{SiO}$ molecules in the supernova have been explored by Liu \& Dalgarno (1994), who suggested that the radiative association of silicon atoms and oxygen atoms to form $\mathrm{SiO}$ is the major mechanism for the formation of $\mathrm{SiO}$ in the supernova. Liu \& Dalgarno (1994) assumed a rate coefficient that is one order of magnitude smaller than the rate coefficient for the radiative association of carbon atoms and oxygen atoms to form CO (Dalgarno, Du, \& You 1990). However, the rate coefficient for the radiative association to form $\mathrm{SiO}$ has now been calculated by Andreazza, Singh, \& Sanzovo (1995). It is a factor of 30 larger than that adopted by Liu \& Dalgarno (1994). Thus, more efficient destruction mechanisms for SiO must be operative in the supernova ejecta than the ionization and dissociation by energetic electron impact that were proposed by Liu \& Dalgarno (1994).

We point out in this paper chemical reactions that destroy $\mathrm{SiO}$ in the ejecta that can balance the more rapid formation of SiO. The presence of argon and neon in the silicon-oxygen region of the ejecta significantly enhances the efficiency of destruction of $\mathrm{SiO}$ in the supernova due to charge transfer reactions with $\mathrm{Ar}^{+}$and $\mathrm{Ne}^{+}$. As a result, the conclusion of our earlier chemistry model (Liu \& Dalgarno 1994) remains valid that the $10^{-4}-10^{-3} M_{\odot}$ of $\mathrm{SiO}$ observed in SN 1987A can be produced by chemistry models in which there is no microscopic mixing of helium into the silicon-oxygen region of the supernova ejecta.

\section{CHEMISTRY OF SiO}

We have explored the chemical processes that form and destroy silicon monoxide molecules in SN 1987A, in addition to those presented in our previous model (Liu \& Dalgarno 1994). The SiO chemistry depends on the elemental composition of the silicon-oxygen region of the supernova ejecta and the degree of mixing of other elements in this region. Dynamical instabilities during and after the supernova explosion cause macroscopic mixing of elements in different layers rather than microscopic mixing. We used unmixed models with the elemental compositions predicted by the explosive nucleosynthesis models of Woosley, Pinto, \& Weaver (1988) and of Thielemann, Hashimoto, \& Nomoto (1990). For the composition of Woosley et al. (1988), $0.14 M_{\odot}$ of silicon and $0.02 M_{\odot}$ of oxygen in the silicon-oxygen region are mixed with $0.06 M_{\odot}$ of sulfur, $0.0093 M_{\odot}$ of argon, and $0.0043 M_{\odot}$ of calcium. The adjacent region, which contains most of the oxygen mass in the supernova, is not expected to be a major site for $\mathrm{SiO}$ formation because neon is the dominant constituent in this region and $\mathrm{Ne}^{+}$is effective in destroying the $\mathrm{SiO}$ molecules even if there is silicon mixed into the region. For the composition of Thielemann et al. (1990), $\mathrm{SiO}$ is likely to be abundant in the region where there is $0.01 M_{\odot}$ of silicon and $0.1 M_{\odot}$ of oxygen together with $0.004 M_{\odot}$ of sulfur, $0.007 M_{\odot}$ of magnesium, and a small amount of carbon. In other regions with significant amounts of silicon, $\mathrm{SiO}$ is not expected to be abundant, either because of the deficiency of oxygen or because of destruction by $\mathrm{Ne}^{+}$and $\mathrm{Ar}^{+}$. To investigate the sensitivity of the chemistry to possible microscopic mixing, we also used the composition in the mixed model of Nomoto et al. (1991) in which $0.085 M_{\odot}$ of silicon and 1.48 $M_{\odot}$ of oxygen are mixed with $0.114 M_{\odot}$ of carbon, 0.147 $M_{\odot}$ of magnesium, $0.229 M_{\odot}$ of neon, $0.0229 M_{\odot}$ of sulfur, $0.00378 M_{\odot}$ of argon, $0.00325 M_{\odot}$ of calcium, $0.073 M_{\odot}$ of iron, and other minor elements.

The chemistry is not sensitive to the temperature, and we assumed that the gas kinetic temperature is the same as the temperature derived by Liu \& Dalgarno (1994) from the rotational level populations of $\mathrm{SiO}$, which is about $2000 \mathrm{~K}$. We assumed that the silicon-oxygen region of the ejecta expanded homologously with the density

$$
n=7 \times 10^{10}(t / 100 \text { days })^{-3} \mathrm{~cm}^{-3},
$$

at time $t$ after the supernova explosion (Liu \& Dalgarno 1994).

\subsection{Formation of $\mathrm{SiO}$}

There was no evidence of dust formation in the ejecta of SN 1987A until about 530 days after the supernova explosion (Lucy et al. 1991). In the absence of grains, molecular formation in the gas phase must have been initiated by radiative processes. Liu \& Dalgarno (1994) suggested that 
the direct radiative association

$$
\mathrm{Si}+\mathrm{O} \rightarrow \mathrm{SiO}+h v
$$

is the most important mechanism for the formation of $\mathrm{SiO}$ molecules in SN 1987A. Its rate coefficient has been calculated by Andreazza et al. (1995) to be $5.52 \times 10^{-18} T^{0.31 \pm 0.02} \mathrm{~cm}^{3} \mathrm{~s}^{-1}$, where $T$ is the temperature in degrees kelvin. The formation of SiO may also be accomplished indirectly by the radiative association

$$
\mathrm{Si}^{+}+\mathrm{O} \rightarrow \mathrm{SiO}^{+}+h v,
$$

with a rate coefficient of $9.7 \times 10^{-19}-7.4 \times 10^{-23} \mathrm{~T}$ $+4.4 \times 10^{-27} T^{2} \mathrm{~cm}^{3} \mathrm{~s}^{-1}$ (Andreazza et al. 1995), followed by charge transfer reactions with metals such as

$$
\begin{aligned}
\mathrm{SiO}^{+}+\mathrm{Si} & \rightarrow \mathrm{Si}^{+}+\mathrm{SiO}, \\
\mathrm{SiO}^{+}+\mathrm{Ca} & \rightarrow \mathrm{Ca}^{+}+\mathrm{SiO} .
\end{aligned}
$$

However, this sequence is an unimportant source of $\mathrm{SiO}$ because $\mathrm{SiO}^{+}$is destroyed predominantly by dissociation recombination

$$
\mathrm{SiO}^{+}+e \rightarrow \mathrm{Si}+\mathrm{O} .
$$

Dissociation recombination proceeds rapidly in the supernova ejecta, which has an ionization fraction of $10^{-2}$. An additional source of $\mathrm{SiO}$ is provided by the radiative association

$$
\mathrm{O}+\mathrm{O} \rightarrow \mathrm{O}_{2}+h v
$$

with a rate coefficient of about $8.5 \times 10^{-21} \mathrm{~cm}^{3} \mathrm{~s}^{-1}$ at 2000 K (Babb \& Dalgarno 1995), followed by the neutral reaction

$$
\mathrm{Si}+\mathrm{O}_{2} \rightarrow \mathrm{SiO}+\mathrm{O},
$$

with a rate coefficient of $2.7 \times 10^{-10} \mathrm{~cm}^{3} \mathrm{~s}^{-1}$ (Husain \& Norris 1978) or $9 \times 10^{-12} \mathrm{~cm}^{3} \mathrm{~s}^{-1}$ (Swearengen, Davies, \& Niemczyk 1978). The sequence initiated by radiative attachment

$$
\begin{aligned}
& e+\mathrm{Si} \rightarrow \mathrm{Si}^{-}+h v, \\
& e+\mathrm{O} \rightarrow \mathrm{O}^{-}+h v,
\end{aligned}
$$

followed by associative detachment

$$
\begin{aligned}
& \mathrm{O}^{-}+\mathrm{Si} \rightarrow \mathrm{SiO}+e, \\
& \mathrm{Si}^{-}+\mathrm{O} \rightarrow \mathrm{SiO}+e,
\end{aligned}
$$

is a negligible path for the formation of $\mathrm{SiO}$ molecules in the ejecta because the negative ions are effectively destroyed by photodetachment

$$
\begin{aligned}
& \mathrm{O}^{-}+h v \rightarrow \mathrm{O}+e, \\
& \mathrm{Si}^{-}+h v \rightarrow \mathrm{Si}+e,
\end{aligned}
$$

and by mutual neutralization such as

$$
\begin{aligned}
\mathrm{Ne}^{+}+\mathrm{O}^{-} & \rightarrow \mathrm{Ne}+\mathrm{O}, \\
\mathrm{Ar}^{+}+\mathrm{Si}^{-} & \rightarrow \mathrm{Ar}+\mathrm{Si} .
\end{aligned}
$$

\subsection{Destruction of $\mathrm{SiO}$}

\subsubsection{Energetic Electron Impact}

The silicon monoxide molecules formed in SN 1987A can be destroyed by impacts with energetic electrons created by the decay of the radioactive nuclei ${ }^{56} \mathrm{Co}$ and ${ }^{57} \mathrm{Co}$. The fast electrons can ionize and dissociate $\mathrm{SiO}$,

$$
\begin{aligned}
e+\mathrm{SiO} & \rightarrow e+\mathrm{SiO}^{+}+e, \\
& \rightarrow e+\mathrm{Si}+\mathrm{O}, \\
& \rightarrow e+\mathrm{Si}^{+}+\mathrm{O}+e, \\
& \rightarrow e+\mathrm{O}^{+}+\mathrm{Si}+e,
\end{aligned}
$$

(Liu \& Dalgarno 1994). The similar processes for carbon monoxide have been analyzed in detail (Liu \& Victor 1994) and applied to the CO chemistry of SN 1987A (Liu, Dalgarno, \& Lepp 1992; Liu \& Dalgarno 1995). We assumed the same rate of destruction by energetic electron impact for $\mathrm{SiO}$ as for $\mathrm{CO}$. For an average energy deposition rate per particle in the oxygen core of

$$
L=60 \exp (-t / \tau)\left\{1-\exp \left[-\phi_{0}\left(t / t_{0}\right)^{-2}\right] v\right\} \mathrm{eV} \mathrm{day}^{-1},
$$

where $\tau=111.26$ days and $\phi_{0}=31.1$ at $t_{0}=100$ days (Liu $\&$ Dalgarno 1995), and for a mean energy per ion pair of about $30 \mathrm{eV}$ for $\mathrm{SiO}$, the rate of destruction of $\mathrm{SiO}$ by energetic electron impact can be estimated to be

$$
R=2 \exp (-t / \tau)\left\{1-\exp \left[-\phi_{0}\left(t / t_{0}\right)^{-2}\right]\right\} \mathrm{day}^{-1} .
$$

Since the radiative association (2) proceeds faster than assumed in our previous chemistry model (Liu \& Dalgarno 1994) in which energetic electron impact is the dominant mechanism for the destruction of SiO in SN 1987A, more efficient destruction mechanisms must exist and will be explored below.

\subsubsection{Photodissociation and Photoionization}

A possible mechanism for the destruction of $\mathrm{SiO}$ in $\mathrm{SN}$ 1987A is provided by photodissociation and photoionization. The ultraviolet photons are produced by excitation of atoms and ions by energetic electron impact and by recombination of ions following ionization by energetic electron impact. These energetic electrons are produced following radioactive decay of ${ }^{56} \mathrm{Co}$ and ${ }^{57} \mathrm{Co}$. An estimate of the ultraviolet flux of $4 \times 10^{15}$ photons $\mathrm{cm}^{-2} \mathrm{~s}^{-1}$ was made by Petuchowski et al. (1989), based on the tentative identification by Moseley et al. (1989) of a spectral feature at $22.9 \mu \mathrm{m}$ as an emission line of $\mathrm{Fe}^{2+}$. Petuchowski et al. (1989) assumed that the relative abundance of $\mathrm{Fe}^{2+} / \mathrm{Fe}^{+}$is controlled by a balance between the photoionization of $\mathrm{Fe}^{+}$ and the charge transfer of $\mathrm{Fe}^{2+}$ with neutral oxygen. About half of the photons ionizing $\mathrm{Fe}^{+}$are energetic enough to destroy SiO.

Even if the identification of the $\mathrm{Fe}^{2+}$ emission line is accepted, no other doubly charged species having been found in the ejecta, the inferred ultraviolet flux is too large because the analysis ignores the ionization caused by energetic electrons. Li, McCray, \& Sunyaev (1993) have shown that the observed infrared emission of $\mathrm{Fe}^{+}$can be accounted for by fast electrons for the first two years, with the ultraviolet photons contributing only at later times. Because the core experienced a considerable expansion driven by the large deposition of energy ( $\mathrm{Li}$ et al. 1993), the density is low. There is little oxygen or elements other than iron, and $\mathrm{Fe}^{2+}$ is removed slowly by radiative and dielectronic recombination and by charge transfer

$$
\mathrm{Fe}^{++}+\mathrm{Fe} \rightarrow \mathrm{Fe}^{+}+\mathrm{Fe}^{+}
$$


to the neutral iron component.

Considerable shielding of any ultraviolet flux by neutral silicon and by $\mathrm{SiO}$ itself is also to be expected so that only the chemistry on the surface of the clumps containing the $\mathrm{Si}$ and the $\mathrm{O}$ will be affected. The optical depth of the silicon contained in a velocity interval as small as $1 \mathrm{~km} \mathrm{~s}^{-1}$ is of the order of $10^{5}$ for a photoionization cross section of $10^{-17}$ $\mathrm{cm}^{2}$ (Conneely, Smith, \& Lipsky 1970; Chapman \& Henry 1972). The mean photodissociation cross section of $\mathrm{SiO}$ is probably of the order of $10^{-18} \mathrm{~cm}^{2}$ so that even with high flux (Petuchowski et al. 1989) the unshielded dissociation rate is only $10^{2}$ day $^{-1}$ and the shielded rate is $10^{-3}$ day $^{-1}$, much lower than the destruction rate due to energetic electron impact.

\subsubsection{Charge Transfer}

In the absence of microscopic mixing of helium in the silicon-oxygen region of the ejecta, the dissociative charge transfer reactions

$$
\begin{aligned}
\mathrm{He}^{+}+\mathrm{SiO} & \rightarrow \mathrm{Si}^{+}+\mathrm{O}+\mathrm{He}, \\
& \rightarrow \mathrm{O}^{+}+\mathrm{Si}+\mathrm{He},
\end{aligned}
$$

do not occur. The reactions

$$
\begin{aligned}
\mathrm{C}^{+}+\mathrm{SiO} & \rightarrow \mathrm{SiO}^{+}+\mathrm{C}, \\
& \rightarrow \mathrm{Si}^{+}+\mathrm{CO}, \\
& \rightarrow \mathrm{CO}^{+}+\mathrm{Si},
\end{aligned}
$$

are unimportant mechanisms for destruction of the $\mathrm{SiO}$ molecules in the supernova even if the carbon comingles with the $\mathrm{SiO}$ because rapid charge transfers to metals such as

$$
\begin{aligned}
\mathrm{C}^{+}+\mathrm{Si} & \rightarrow \mathrm{Si}^{+}+\mathrm{C}, \\
\mathrm{C}^{+}+\mathrm{Ca} & \rightarrow \mathrm{Ca}^{+}+\mathrm{C},
\end{aligned}
$$

cause the carbon to be mostly neutral in the ejecta. An important mechanism for the destruction of the $\mathrm{SiO}$ molecules in SN 1987A is provided by the charge transfer reactions

$$
\begin{aligned}
\mathrm{Ar}^{+}+\mathrm{SiO} & \rightarrow \mathrm{SiO}^{+}+\mathrm{Ar}, \\
\mathrm{Ne}^{+}+\mathrm{SiO} & \rightarrow \mathrm{SiO}^{+}+\mathrm{Ne}, \\
& \rightarrow \mathrm{Si}^{+}+\mathrm{O}+\mathrm{Ne} .
\end{aligned}
$$

Silicon and argon together with sulfur and calcium are the main products of oxygen burning during the explosive nucleosynthesis, and they are intermixed together in the supernova ejecta. Neon is produced in the stellar nucleosynthesis during the main sequence, and neon and oxygen are mixed microscopically in the supernova ejecta. The substantial presence of argon and neon in the silicon-oxygen region significantly enhances the destruction efficiency for the SiO molecules in SN 1987A. Reactions (31)-(33) should proceed more rapidly than the corresponding reactions of $\mathrm{CO}$ :

$$
\begin{aligned}
& \mathrm{Ar}^{+}+\mathrm{CO} \rightarrow \mathrm{CO}^{+}+\mathrm{Ar}, \\
& \mathrm{Ne}^{+}+\mathrm{CO} \rightarrow \mathrm{CO}^{+}+\mathrm{Ne},
\end{aligned}
$$

because $\mathrm{SiO}$ is less stable than $\mathrm{CO}$. The rate coefficient for reaction (34) has been measured to be about $4 \times 10^{-11} \mathrm{~cm}^{3}$ $\mathrm{s}^{-1}$ at the center-of-mass kinetic energy of $0.1 \mathrm{eV}$ (Rebrion, Rowe, \& Marquette 1989; Flesch, Nourbakhsh, \& Ng
1991). Reaction (35) proceeds at a rate coefficient of $2 \times 10^{-12} \mathrm{~cm}^{3} \mathrm{~s}^{-1}$ at $0.2 \mathrm{eV}$, and the rate coefficient increases rapidly as the kinetic energy increases (Jones et al. 1981; Villinger et al. 1983). We assumed rate coefficients of $4 \times 10^{-11} \mathrm{~cm}^{3} \mathrm{~s}^{-1}$ and $2 \times 10^{-12} \mathrm{~cm}^{3} \mathrm{~s}^{-1}$ for reactions (31) and (32)-(33), respectively.

The efficiency of $\mathrm{Ar}^{+}$and $\mathrm{Ne}^{+}$in destroying $\mathrm{SiO}$ depends directly on the ionization fractions of argon and neon in the silicon-oxygen region of the supernova ejecta, which are controlled by charge transfer reactions of $\mathrm{Ar}^{+}$and $\mathrm{Ne}^{+}$ with neutral atoms. Charge transfer reactions involving noble ions are likely to be slow. The charge transfer reaction

$$
\mathrm{He}^{+}+\mathrm{C} \rightarrow \mathrm{C}^{+}+\mathrm{He}
$$

proceeds slowly, with a rate coefficient of $5.2 \times 10^{-14} \mathrm{~cm}^{3}$ $\mathrm{s}^{-1}$ at $2000 \mathrm{~K}$ (Kimura et al. 1993). The rate coefficient for the charge transfer reaction,

$$
\mathrm{He}^{+}+\mathrm{O} \rightarrow \mathrm{O}^{+}+\mathrm{He},
$$

is negligibly small (Kimura et al. 1994). We assumed that charge transfer reactions such as

$$
\begin{aligned}
& \mathrm{Ar}^{+}+\mathrm{Si} \rightarrow \mathrm{Si}^{+}+\mathrm{Ar}, \\
& \mathrm{Ar}^{+}+\mathrm{O} \rightarrow \mathrm{O}^{+}+\mathrm{Ar}, \\
& \mathrm{Ne}^{+}+\mathrm{Si} \rightarrow \mathrm{Si}^{+}+\mathrm{Ne}, \\
& \mathrm{Ne}^{+}+\mathrm{O} \rightarrow \mathrm{O}^{+}+\mathrm{Ne},
\end{aligned}
$$

are slow, with rate coefficients in the range around $10^{-13}$ $\mathrm{cm}^{3} \mathrm{~s}^{-1}$ so that the ionization fractions of argon and neon are high, and we assumed that charge transfer reactions such as

$$
\mathrm{O}^{+}+\mathrm{Si} \rightarrow \mathrm{Si}^{+}+\mathrm{O}
$$

are rapid with rate coefficients of $10^{-9} \mathrm{~cm}^{3} \mathrm{~s}^{-1}$.

\section{MASS OF SiO IN SN 1987A}

Shown in Figure 1 and listed in Table 1 are the masses of $\mathrm{SiO}$ in SN 1987A derived from the fundamental emission spectra of $\mathrm{SiO}$ by taking into account optical depth effects

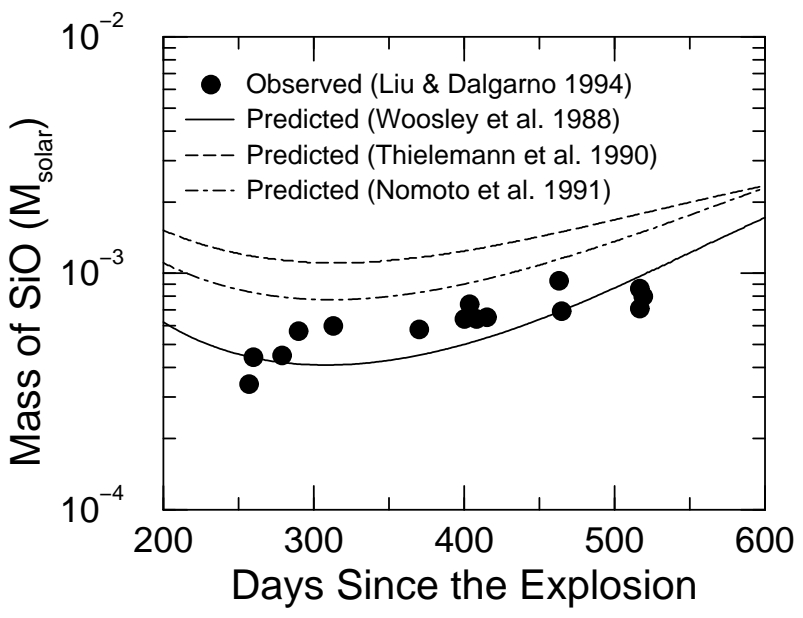

FIG. 1.- The SiO mass in SN 1987A as a function of time since the supernova explosion. The closed circles are the observed masses derived by Liu \& Dalgarno (1994) from the fundamental emission spectra of $\mathrm{SiO}$ by taking into account the optical depth effects and departures from local thermodynamic equilibrium. The solid, dashed, and dot-dashed lines are the predictions of the chemistry for the elemental compositions of Woosley et al. (1988), Thielemann et al. (1990), and Nomoto et al. (1991), respectively. 
TABLE 1

MASS OF SiO IN SN 1987A

\begin{tabular}{|c|c|c|c|c|}
\hline \multirow[b]{2}{*}{$\begin{array}{l}\text { EPOCH } \\
\text { (days) }\end{array}$} & \multirow{2}{*}{  } & \multicolumn{3}{|c|}{ PREDICTED Mass FROM CHEMistry } \\
\hline & & $\begin{array}{l}\text { Woosley et al. } \\
\left(10^{-4} M_{\odot}\right)\end{array}$ & $\begin{array}{l}\text { Thielemann et al. } \\
\qquad\left(10^{-4} M_{\odot}\right)\end{array}$ & $\begin{array}{l}\text { Nomoto et al. } \\
\left(10^{-4} M_{\odot}\right)\end{array}$ \\
\hline $257 \ldots \ldots$ & 3.4 & 4.4 & 11.9 & 8.4 \\
\hline $260 \ldots \ldots$ & 4.4 & 4.4 & 11.8 & 8.3 \\
\hline $279 \ldots \ldots$ & 4.5 & 4.2 & 11.4 & 7.9 \\
\hline $290 \ldots \ldots$ & 5.7 & 4.1 & 11.2 & 7.8 \\
\hline $313 \ldots \ldots$ & 6.0 & 4.1 & 11.1 & 7.7 \\
\hline $370 \ldots \ldots$ & 5.8 & 4.5 & 11.7 & 8.3 \\
\hline $400 \ldots \ldots$ & 6.4 & 5.0 & 12.4 & 9.0 \\
\hline $404 \ldots \ldots$ & 7.4 & 5.1 & 12.6 & 9.1 \\
\hline $408 \ldots \ldots$ & 6.4 & 5.2 & 12.7 & 9.2 \\
\hline $415 \ldots \ldots$ & 6.5 & 5.3 & 12.9 & 9.5 \\
\hline $463 \ldots \ldots$ & 9.3 & 6.9 & 14.9 & 11.5 \\
\hline $465 \ldots \ldots$ & 6.9 & 7.0 & 15.0 & 14.6 \\
\hline $517 \ldots \ldots$ & 7.1 & 9.7 & 17.8 & 14.8 \\
\hline $517 \ldots \ldots$ & 8.6 & 9.7 & 17.8 & 14.8 \\
\hline $519 \ldots \ldots$ & 8.0 & 9.9 & 18.0 & 15.0 \\
\hline
\end{tabular}

and departures from local thermodynamic equilibrium (Liu \& Dalgarno 1994). The observed $\mathrm{SiO}$ masses increase significantly from 257 days to 465 days, followed by a slight decrease to 519 days. The absence of $\mathrm{SiO}$ emission after 530 days (Lucy et al. 1991) indicates that the $\mathrm{SiO}$ molecules were depleted into dust grains for which the $\mathrm{SiO}$ molecules themselves may have provided the seed.

These observed masses of $\mathrm{SiO}$ are in good agreement with the masses of $\mathrm{SiO}$ predicted for the various supernova models that are shown in Figure 1 and listed in Table 1. Similar to the temporal behavior of the observed $\mathrm{SiO}$ masses, the predicted $\mathrm{SiO}$ masses increase significantly after about 300 days. They depend on the elemental composition. The observed $\mathrm{SiO}$ masses are best reproduced by the chemistry model based on the composition of Woosley et al. (1988), for which the observed and predicted $\mathrm{SiO}$ masses agree within a factor of 1.5 . The $\mathrm{SiO}$ masses predicted by the chemistry models with the compositions of Thielemann et al. (1990) and Nomoto et al. (1991) overestimate the observed $\mathrm{SiO}$ masses by factors of no more than 3.5. Thus, the chemistry is robust and not very sensitive to the elemental composition.

The predicted minimum $\mathrm{SiO}$ mass of $4 \times 10^{-4} M_{\odot}$ at about 300 days for the composition of Woosley et al. (1988) is lower by a factor of about 2 than the previous estimate of $7 \times 10^{-4} M_{\odot}$ (Liu \& Dalgarno 1994). The $\mathrm{SiO}$ is formed mainly by the radiative association (2) and is destroyed mainly by the charge transfer reaction (31) with $\mathrm{Ar}^{+}$. Although the rate coefficient of the radiative association (2) is about 30 times higher than assumed in our previous model, the $\mathrm{SiO}$ formation rate is enhanced by an effective factor of only about 4 because the oxygen mass in the SiOforming region is only $\frac{1}{7}$ of the total oxygen mass in the supernova, most of it being in the region in which neon is the dominant element and the charge transfer reactions (32)-(33) limit the abundance of $\mathrm{SiO}$. The presence of argon enhances the $\mathrm{SiO}$ destruction rate by a factor of about 7 through the charge transfer reaction (31). The significant increase at late times in the predicted $\mathrm{SiO}$ mass occurs because the destruction of $\mathrm{SiO}$ by $\mathrm{Ar}^{+}$becomes slower as the result of the rapid decrease in the fractional abundance of $\mathrm{Ar}^{+}$. The loss of $\mathrm{Ar}^{+}$is dominated at early times by the charge transfer reactions (38)-(39), with $\mathrm{Si}$ and $\mathrm{O}$ and accelerated at late times by the charge transfer reaction (31) with $\mathrm{SiO}$ as the fractional abundance of $\mathrm{SiO}$ increases.

For the compositions of Thielemann et al. (1990) and Nomoto et al. (1991), the chemistry models predict about $1 \times 10^{-3} M_{\odot}$ and $8 \times 10^{-4} M_{\odot}$, respectively, for the $\mathrm{SiO}$ masses at 300 days.

The predicted $\mathrm{SiO}$ masses would be reduced by orders of magnitude were the $\mathrm{SiO}$ molecules mixed microscopically with helium because the destruction of $\mathrm{SiO}$ by the dissociative charge transfer reactions $(24)-(25)$ is extremely fast. Thus, the $\mathrm{SiO}$ chemistry permits no microscopic mixing of helium in the silicon-oxygen region where $\mathrm{SiO}$ is abundant.

These additional destruction mechanisms for $\mathrm{SiO}$ in $\mathrm{SN}$ 1987A through the charge transfer reactions (31)-(33) have no effect on the chemistry of CO (Liu et al. 1992; Liu \& Dalgarno 1995) because the argon and neon do not mix into the carbon-oxygen region of the supernova ejecta where the $\mathrm{CO}$ molecules are formed.

This work was supported by the National Science Foundation, Division of Astronomical Sciences, under grant AST 93-01099.

\section{REFERENCES}

Aitken, D. K., Smith, C. H., James, S. D., Roche, P. F., Hyland, A. R., \& McCregor, P. J. 1988, MNRAS, 231, 7P

Andreazza, C. M., Singh, P. D., \& Sanzovo, G. C. 1995, ApJ, 451, 889

Babb, J. F., \& Dalgarno, A. 1995, Phys. Rev. A, 51, 3021

Chapman, R. D. \& Henry, R. J. W. 1972, ApJ, 173, 243

Conneely, M. J., Smith, K, \& Lipsky, L. 1970, J. Phys. B, 3, 493

Dalgarno, A., Du, M. L., \& You, J. H. 1990, ApJ, 349, 675

Flesch, G. D., Nourbakhsh, S., \& Ng, C. Y. 1991, J. Chem. Phys., 95, 3381

Husain, D., \& Norris, P. E. 1978, J. Chem. Soc. Faraday Trans. II, 71, 525
Jones, T. T., Villinger, J., Lister, D. G.,Tichy, M., Birkinshaw, K., \& Twiddy, N. D. 1981, J. Phys. B, 14, 2719

Kimura, M., Dalgarno, A., Chantranupong, L., Li, Y., Hirsch, G., \& Buenker, R. J. 1993, ApJ, 417, 812

Kimura, M., Gu, J. P., Liebermann, H. P., Li, Y., Hirsch, G., \& Buenker, R. J., 1994, Phys. Rev. A, 50, 4854

Li, H., McCray, R., \& Sunyaev, R. A. 1993, ApJ, 419, 824

Liu, W., \& Dalgarno, A. 1994, ApJ, 428, 769 .1995, ApJ, 454, 472 
Liu, W., Dalgarno, A., \& Lepp, S. 1992, ApJ, 396, 679

Liu, W., \& Victor, G. A. 1994, ApJ, 435, 909

Lucy, L. B., Danziger, I. J., Gouiffes, C., \& Bouchet, P. 1991, in Supernovae, ed. S. E. Woosley (New York: Springer), 82

Moseley, S. H., Dwek, E., Silverberg, R. F., Glaccum, W. J., Graham, J. R. \& Loewenstein, R. F. 1989, ApJ, 347, 1119

Nomoto, K., Shigeyama, T., Kumagai, S., \& Yamaoka, H. 1991, in Supernovae, ed. S. E. Woosley (New York: Springer), 176

Petuchowski, S. J., Dwek, E., Allen, J. E., \& Nuth, J. A. 1989, ApJ, 342, 406

Rebrion, C., Rowe, B. R., \& Marquette, J. B. 1989, J. Chem. Phys., 91, 6142
Roche, P. F., Aitken, D. K., Smith, C. H., \& James, S. D. 1989, Nature, 337, 533

Swearengen, P. M., Davies, S. J., \& Niemczyk, T. N. 1978, Chem. Phys Lett., 55, 274

Thielemann, F.-K., Hashimoto, M., \& Nomoto, K. 1990, ApJ, 349, 222

Villinger, H., Futrell, J. H., Richter, R., Saxer, A., Niccolini, St., \& Lindinger, W. 1983, Int. J. Mass Spectrom. Ion Phys., 47, 175

Woosley, S. E., Pinto, P. A., \& Weaver 1988, Proc. Astron. Soc. Australia 7,355 